\title{
An elementary approach to electromagnetic momentum in matter
}

\author{
Rodrigo Medina 1 , 困 and J Stephany, 田 \\ ${ }^{1}$ Centro de Física, Instituto Venezolano de Investigaciones Científicas, Apartado 20632 Caracas 1020-A, Venezuela. \\ ${ }^{2}$ Departamento de Fúsica, Sección de Fenómenos Ópticos, Universidad \\ Simón Bolivar, Apartado Postal 89000, Caracas 1080-A, Venezuela.
}

\begin{abstract}
We present an elementary discussion of the momentum transferred to a conducting sheet by an electromagnetic wave propagating in a polarizable medium. We show that conservation of momentum is consistent with Minkowski's expression for the momentum density.

PACS numbers: $42.50 . \mathrm{Wk}, 45.20 . \mathrm{df}$
\end{abstract}

\section{INTRODUCTION}

Although the conservation of energy and momentum of electromagnetic fields in vacuum is a very well understood problem, inside a polarizable medium it is still a matter of discussion. A review of the literature on this subject can be found in [1].

In the vacuum, Poynting's equation for the exchange of energy between the field and free charges is easily derived starting from Lorentz's force density on charges and currents

$$
\mathbf{f}=\rho \mathbf{E}+\mathbf{j} \times \mathbf{B}
$$

and from the power density on the currents

$$
\dot{w}=\mathbf{j} \cdot \mathbf{E} .
$$

The resulting equation

$$
\frac{\partial u}{\partial t}+\nabla \cdot \mathbf{S}=-\mathbf{j} \cdot \mathbf{E}
$$

written in terms of

$$
u=u_{\mathrm{v}}=\frac{1}{2} \epsilon_{0} E^{2}+\frac{1}{2 \mu_{0}} B^{2}
$$

and

$$
\mathbf{S}=\frac{1}{\mu_{0}} \mathbf{E} \times \mathbf{B}
$$

is an identity valid for any solution of Maxwell's equations. It is interpreted as a local energy conservation equation by taking $u_{\mathrm{v}}$ as the energy density of the electromagnetic field and Poynting's vector $\mathbf{S}$ as the energy flux current vector. The power exerted by the matter on the fields is assumed to be the opposite of (2).

Electromagnetic fields also carry momentum. The derivation of the equation for the momentum exchange

\footnotetext{
* rmedina@ivic.gob.ve

† stephany@usb.ve
}

requires the use of Maxwell's stress tensor and a somewhat more involved calculation which do not make easy an elementary presentation of the subject. In many textbooks (e.g [2], 3]) radiation pressure is simply introduced as a new fact, sometimes referring to it as one of Maxwell's achievements. In the vacuum the momentum density is accepted to be

$$
\mathbf{g}=\epsilon_{0} \mathbf{E} \times \mathbf{B}=\frac{1}{c^{2}} \mathbf{S} .
$$

which leads to the relation

$$
\Delta p=\frac{\Delta U}{c}
$$

for the energy and momentum that an electromagnetic wave releases when absorbed. Because of this in other elementary presentations (e.g [4]) the density of momentum (6) is justified by working on the analogy with a relativistic particle, whose momentum and energy are related by

$$
\mathbf{p}=\frac{E}{c^{2}} \mathbf{v} .
$$

For an ultra relativistic particle $(v \rightarrow c)$ holds

$$
\mathbf{p}=\frac{E}{c} \hat{\mathbf{v}} .
$$

By assuming that this last relation is also valid for a packet of electromagnetic waves traveling in some direction, one gets (7) and therefore $g=c^{-2} S$ in accord with (6). This procedure has the shortcoming of having a non electromagnetic element, the relativistic particle, introduced in the discussion.

A more enlighting treatment of the subject is the one presented for example in Ref. [5]. There, the interaction of electromagnetic wave packets coming from vacuum with electric currents in a non ideal conductor are considered. The impulse transferred to the currents is computed and showed to be consistent with (6) and (7). In this paper we show that this same idea can also be applied to the propagation of light in matter.

As we already commented, what happens with momentum inside matter is still not completely clear from the 
theoretical point of view. For the energy, Poynting [6] proposed that Eq. (3) remains valid with

$$
u=\frac{1}{2} \mathbf{D} \cdot \mathbf{E}+\frac{1}{2} \mathbf{H} \cdot \mathbf{B}
$$

and

$$
\mathbf{S}=\mathbf{E} \times \mathbf{H} .
$$

Here, $\mathbf{j}$ is the free current density, $\mathbf{D}=\epsilon_{0} \mathbf{E}+\mathbf{P}$ is the electric displacement and $\mathbf{H}=1 / \mu_{0} \mathbf{B}-\mathbf{M}$ is the magnetizing field. It may be shown that Eq.(3) is consistent with Maxwell's equations only for materials with linear polarizations, $\mathbf{P} \propto \mathbf{E}$ and $\mathbf{M} \propto \mathbf{B}$. In particular it holds for a material with permittivity $\epsilon$ and permeability $\mu$, $\mathbf{P}=\left(\epsilon-\epsilon_{0}\right) \mathbf{E}$ and $\mathbf{M}=\left[\left(\mu-\mu_{0}\right) / \mu_{0} \mu\right] \mathbf{B}$, but it also holds in the more general situation of a non-isotropic material.

Poynting's density is the vacuum energy density $u_{\mathrm{v}}$ plus terms that depend on the polarization $\mathbf{P}$ and the magnetization $\mathbf{M}$,

$$
u=u_{\mathrm{v}}+\frac{1}{2} \mathbf{P} \cdot \mathbf{E}-\frac{1}{2} \mathbf{M} \cdot \mathbf{B} .
$$

The electric term corresponds to the energy density of deformation of matter as it develops electric dipole moments. The magnetic term is the work density made on microscopic currents by the induced electric field. The work done on electric and magnetic moments is already included in Poynting's energy density, that is the reason why in the right hand side of equation (3) only the power of the free current density appears. In other words, for an electromagnetic wave in a material medium, Poynting's expression corresponds to the energy density of the whole wave, including the change of energy of matter as the wave propagates in the medium.

For the momentum density of the fields in matter the situation is less clear. There is a dispute that has lasted more than a century [1] on the subject. Minkowski [7] proposed

$$
\mathbf{g}_{\mathrm{Min}}=\mathbf{D} \times \mathbf{B},
$$

which for individual photons corresponds to using (9) replacing $c$ by the speed of light in matter $v=(\epsilon \mu)^{-1 / 2}$. This was challenged by Abraham [8, 9] who proposed instead

$$
\mathbf{g}_{\mathrm{Abr}}=\frac{1}{c^{2}} \mathbf{E} \times \mathbf{H}=\frac{1}{c^{2}} \mathbf{S},
$$

which for photons corresponds to using directly (8).

To shed some light on this issue, in this paper we study a simple example that can be solved exactly. It consists of a packet of plane waves propagating in a material with permeability $\mu$ and permittivity $\epsilon$. The medium being homogeneous there is no refraction and the wave travels conserving momentum and energy. Inside the material there is a flat thin film of conducting material in which
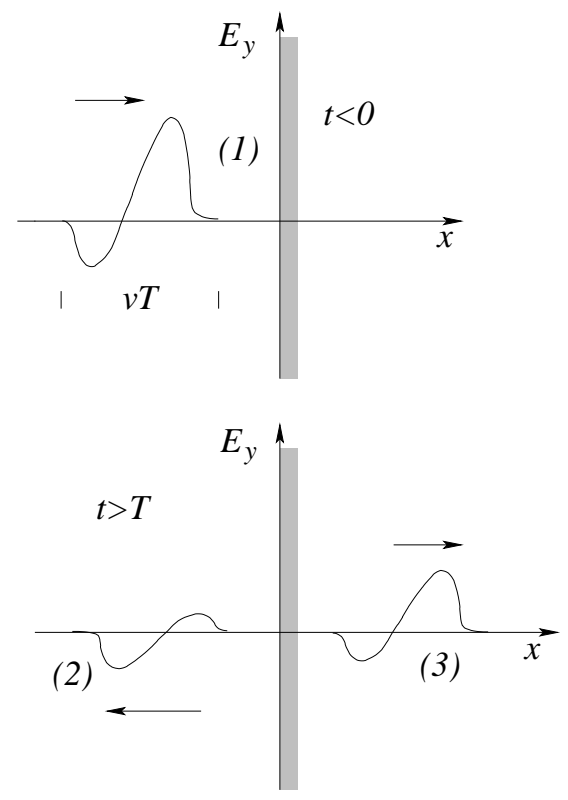

FIG. 1. Incident (1), reflected (2) and transmitted (3) packets

Ohm's law holds, $\mathbf{j} \propto \mathbf{E}$. When the wave packet falls on the film it splits in reflected and transmitted packets, whose amplitudes are fixed by the boundary conditions (See Figure 11). The total work done on the currents in the conducting film can be calculated using (2). The result is consistent with Poynting's energy density (10). Also, the impulse on the currents of the film can be calculated using (11). We found that the result is consistent with momentum conservation only if the momentum carried by the waves is given by Minkowski's expression (13)

\section{MOMENTUM TRANSFER}

Consider a medium with electric and magnetic permeabilities $\epsilon$ and $\mu$ filling the space. At the plane $x=0$ there is a sheet of thickness $a$ made of a conductive material of resistivity $\rho$. A packet of electromagnetic plane waves polarized in the direction $y$ is traveling in the $x$ direction. Suppose it has a spatial length $v T$ with $v=1 / \sqrt{\epsilon \mu}, T$ a time scale, and a wave length $\lambda$ such that $a<<\lambda$ and $a<<v T$. In terms of a suitable function $h(t)$ which vanishes outside the interval $[0, T]$ the incident wave is,

$$
\begin{aligned}
& \mathbf{E}_{1}(x, y, z, t)=E_{1} h(t-x / v) \theta(-x) \hat{\mathbf{y}} \quad x<0 \\
& \mathbf{B}_{1}(x, y, z, t)=\frac{1}{v} E_{1} h(t-x / v) \theta(-x) \hat{\mathbf{z}} .
\end{aligned}
$$

The reflected and transmitted waves are,

$$
\begin{aligned}
& \mathbf{E}_{2}(x, y, z, t)=E_{2} h(t+x / v) \theta(-x) \hat{\mathbf{y}} \\
& \mathbf{B}_{2}(x, y, z, t)=-\frac{1}{v} E_{2} h(t+x / v) \theta(-x) \hat{\mathbf{z}}
\end{aligned}
$$




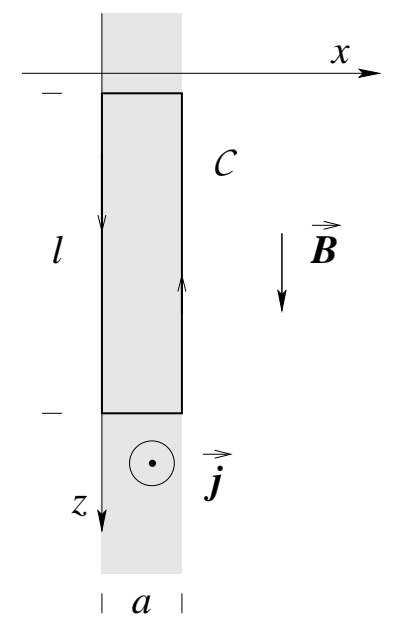

FIG. 2. The integration path

and

$$
\begin{aligned}
& \mathbf{E}_{3}(x, y, z, t)=E_{3} h(t-x / v) \theta(x) \hat{\mathbf{y}}, \\
& \mathbf{B}_{3}(x, y, z, t)=\frac{1}{v} E_{3} h(t-x / v) \theta(x) \hat{\mathbf{z}} .
\end{aligned}
$$

At $x=0 \mathbf{E}$ is continuous, $\mathbf{E}\left(0^{+}, y, z, t\right)=\mathbf{E}\left(0^{-}, y, z, t\right)$ implying

$$
E_{1}+E_{2}=E_{3}
$$

There is an electrical current in the sheet. The magnetic field is discontinuous at $x=0$. Taking a rectangular path of length $l$ in the $z$ direction and width $a$ in the $x$ direction we have,

$$
\oint_{C} \mathbf{H} \cdot d \mathbf{l}=\int_{S} \mathbf{j} \cdot d \mathbf{S}+\frac{d}{d t} \int_{S} \mathbf{D} \cdot d \mathbf{S}
$$

where $S$ is the plane surface with boundary $C$ (See Figure 2]). The second integral vanishes because the width of the surface is negligible. The current density is

$$
\mathbf{j}=\frac{1}{\rho} \mathbf{E}=\frac{1}{\rho} E_{3} h(t) \hat{\mathbf{y}} .
$$

Substituting this in the previous equation we have

$$
\frac{1}{\mu}\left(B_{1}-B_{2}-B_{3}\right) h(t) l=j a l
$$

and

$$
\frac{1}{v}\left(E_{1}-E_{2}-E_{3}\right)=\frac{\mu a E_{3}}{\rho} .
$$

Introducing

$$
b=\frac{\mu a v}{2 \rho}
$$

and solving the system we have,

$$
E_{2}=-\frac{b E_{1}}{1+b} \quad, \quad E_{3}=\frac{E_{1}}{1+b} .
$$

The energy of the wave is given by Poynting's expression

$$
u=\frac{1}{2}\left(\epsilon E^{2}+\frac{1}{\mu} B^{2}\right)=\epsilon E^{2} .
$$

For $t<0$ we consider a cylindrical piece of the incident packet with axis parallel to $x$ and cross section $A$. The energy is,

$$
\begin{aligned}
u & =\epsilon E_{1}^{2} h^{2}(t-x / v), \\
U_{1} & =\int \epsilon E_{1}^{2} h^{2}(t-x / v) d^{3} r=\epsilon E_{1}^{2} A v \bar{T}
\end{aligned}
$$

where

$$
\bar{T}=\int_{0}^{T} h^{2}(t) d t
$$

For $t>T$ the energies of the reflected and transmitted waves are,

$$
\begin{aligned}
& U_{2}=\epsilon E_{2}^{2} A v \bar{T}=\left[\frac{b}{1+b}\right]^{2} U_{1}, \\
& U_{3}=\epsilon E_{3}^{2} A v \bar{T}=\left[\frac{1}{1+b}\right]^{2} U_{1} .
\end{aligned}
$$

The power density on the current is

$$
\dot{w}=\mathbf{j} \cdot \mathbf{E}=\frac{E^{2}(t)}{\rho}=\frac{E_{3}^{2} h^{2}(t)}{\rho} .
$$

The total power transferred by the wave is,

$$
\frac{d W}{d t}=\int \mathbf{E} \cdot \mathbf{j} d^{3} r=\frac{E_{3}^{2} A a h(t)^{2}}{\rho}
$$

and the work on the current is

$$
W=\int \frac{d W}{d t} d t=\frac{E_{3}^{2} A a \bar{T}}{\rho}=\frac{2 b}{[1+b]^{2}} U_{1} .
$$

The energy is conserved

$$
U_{1}=U_{2}+U_{3}+W
$$

Let us now discuss momentum conservation for this system. We compute first the force applied on the sheet. The force density on the current is

$$
\mathbf{f}=\mathbf{j} \times \mathbf{B}
$$

In the approximation where $a$ is infinitesimal, the current density in the sheet, $\mathbf{j}$, is constant because the electric field is continuous. The magnetic field then varies linearly between its values at both sides of the sheet. In computing the total force one has to take the average on the sheet volume. For $0 \leq t \leq T$ we have

$$
\begin{aligned}
\mathbf{F} & =\int \mathbf{f} d^{3} r=j A \int_{0}^{a} B_{z}(x) d x \hat{\mathbf{x}} \\
& =j A a \frac{\left(B_{z}\left(0^{-}\right)+B_{z}\left(0^{+}\right)\right)}{2} \hat{\mathbf{x}}
\end{aligned}
$$


where

$$
\begin{aligned}
\frac{1}{2}\left(B_{z}\left(0^{-}\right)+B_{z}\left(0^{+}\right)\right) & =\frac{h(t)}{2 v}\left[E_{1}-E_{2}+E_{3}\right] \\
& =\frac{h(t)}{v} E_{1}
\end{aligned}
$$

Substituting this in (39) and using (23) and (27) we get

$$
\mathbf{F}=\frac{A a h^{2}(t) E_{1}^{2}}{v \rho(1+b)} \hat{\mathbf{x}}
$$

The impulse applied to the current sheet is

$$
\begin{aligned}
\mathbf{I} & =\int \mathbf{F} d t=\frac{A a \bar{T} E_{1}^{2}}{v \rho(1+b)} \hat{\mathbf{x}} \\
& =\frac{2 b}{(1+b) v} U_{1} \hat{\mathbf{x}}
\end{aligned}
$$

The momentum carried by the wave is directed in the same direction in which the wave propagates, that is, the direction of $\mathbf{E} \times \mathbf{B}$. The momentum density of the wave may be written in the form,

$$
\mathbf{g}=\alpha \mathbf{E} \times \mathbf{B}
$$

with $\alpha$ a constant to be determined. For $\alpha=\epsilon$ the momentum density is Minkowski's and for $\alpha=1 / c^{2} \mu$ it is Abraham's. Then, the momentum carried by the incident wave is

$$
\begin{aligned}
\mathbf{p}_{1} & =\int \mathbf{g}_{1} d V=\frac{\alpha E_{1}^{2}}{v} \int h^{2}(t-x / v) \hat{\mathbf{x}} d^{3} r \\
& =\alpha E_{1}^{2} A \bar{T} \hat{\mathbf{x}}=\frac{\alpha U_{1}}{\epsilon v} \hat{\mathbf{x}}
\end{aligned}
$$

Analogously

$$
\mathbf{p}_{2}=-\frac{\alpha U_{2}}{\epsilon v} \hat{\mathbf{x}} \quad, \quad \mathbf{p}_{3}=\frac{\alpha U_{3}}{\epsilon v} \hat{\mathbf{x}} .
$$

For the balance equation we get,

$$
\mathbf{p}_{1}-\mathbf{p}_{2}-\mathbf{p}_{3}=\frac{\alpha}{\epsilon v}\left(U_{1}+U_{2}-U_{3}\right) \hat{\mathbf{x}}=\frac{\alpha}{\epsilon} \mathbf{I} .
$$

Momentum is conserved if $\alpha=\epsilon$. The momentum density (43) turns out to be Minkowski's expression,

$$
\mathbf{g}=\mathbf{g}_{\mathrm{Min}}=\mathbf{D} \times \mathbf{B}
$$

The key in the previous argument is the factor $v^{-1}$ that appears in the impulse (42). This can be traced back to the magnetic force in Lorentz's force (11) and the relationship between the magnetic and electric amplitudes in electromagentic waves. This relation is a consequence of Maxwell's equations. For obtaining Abraham's result the factor should have been $c^{-2} v$.

\section{CONCLUSION}

The calculation presented in this paper assumes the validity of Maxwell's equations and Lorentz's force density on the conduction current. Using the properties of the electromagnetic wave solutions of Maxwell's equations in a simple setup we showed that momentum conservation requires the use of Minkowski's expression for the momentum density of the electromagnetic field in a linear medium.

Although the use of the explicit solution of Maxwell equations with boundary conditions puts our approach a little ahead of most introductory physics university courses it is still a very simple and elementary construction which allows the discussion of electromagnetic momentum in material media in a self-consistent way. In particular it also may be used to treat the case when the wave propagates in the vacuum.
[1] D. J. Griffiths, Am. J. Phys. 80, 7 (2012).

[2] D. Halliday, R. Resnik and J. Walker,Fundamentals of Physics, 10th Edition, John Wiley and Sons, New York, 2014, p. 983.

[3] H. D. Young and R. A. Freedman, Sears and Zemansky's University Physics, 13th Edition, Addison-Wesley, Boston, 2012, p. 1068.

[4] M. Alonso and E. J. Finn, Physics, Addison-Wesley, New
York, 1992, p. 788.

[5] D. Halliday and R. Resnik, Physics, Part two, 3d Edition, John Wiley and Sons, New York, 1978, p. 921.

[6] J. H. Poynting, Phil. Trans. R. Soc. 175, 343-361 (1884).

[7] H. Minkowski, Nachr. Ges. Wiss. Göttingen, 53, (1908).

[8] M. Abraham, Rend. Circ. Mat. Palermo 28, 1-28, (1909).

[9] M. Abraham, Rend. Circ. Mat. Palermo 30, 33i-46, (1910). 